\title{
Estimation of multivariate detection limits of four quality parameters in licorice using MEMS-NIR spectrometry coupled with two sampling accessories
}

\author{
Zhisheng $\mathrm{Wu}^{*}+$, Xinyuan Shi, Na Zhao, Yanling Pei, Manfei Xu, \\ Luwei Zhou, Yang Li and Yanjiang Qiao ${ }^{\dagger+*}$ \\ Beijing University of Chinese Medicine, P. R. China 100102 \\ Beijing Key Laboratory for Basic and Development \\ Research on Chinese Medicine \\ Beijing, P. R. China 100102 \\ Key Laboratory of TCM-information Engineer \\ of State Administration of TCM \\ Beijing, P. R. China 100102 \\ *wzs@bucm.edu.cn \\ †yjqiao@263.net
}

Received 8 April 2014

Accepted 29 June 2014

Published 6 August 2014

\begin{abstract}
In this work, multivariate detection limits (MDL) estimator was obtained based on the microelectro-mechanical systems-near infrared (MEMS-NIR) technology coupled with two sampling accessories to assess the detection capability of four quality parameters (glycyrrhizic acid, liquiritin, liquiritigenin and isoliquiritin) in licorice from different geographical regions. 112 licorice samples were divided into two parts (calibration set and prediction set) using KennardStone (KS) method. Four quality parameters were measured using high-performance liquid chromatography (HPLC) method according to Chinese pharmacopoeia and previous studies. The MEMS-NIR spectra were acquired from fiber optic probe (FOP) and integrating sphere, then the partial least squares (PLS) model was obtained using the optimum processing method. Chemometrics indicators have been utilized to assess the PLS model performance. Model assessment using chemometrics indicators is based on relative mean prediction error of all concentration levels, which indicated relatively low sensitivity for low-content analytes (below 1000 parts per million (ppm)). Therefore, MDL estimator was introduced with alpha error and beta error based on good prediction characteristic of low concentration levels. The result suggested that MEMSNIR technology coupled with fiber optic probe (FOP) and integrating sphere was able to detect minor analytes. The result further demonstrated that integrating sphere mode (i.e., $\mathrm{MDL}_{0.05,0.05}$, $0.22 \%$ ) was more robust than FOP mode (i.e., $\mathrm{MDL}_{0.05,0.05}, 0.48 \%$ ). In conclusion, this research
\end{abstract}

\$Corresponding authors.

This is an Open Access article published by World Scientific Publishing Company. It is distributed under the terms of the Creative Commons Attribution 3.0 (CC-BY) License. Further distribution of this work is permitted, provided the original work is properly cited. 
proposed that MDL method was helpful to determine the detection capabilities of low-content analytes using MEMS-NIR technology and successful to compare two sampling accessories.

Keywords: Near-infrared spectrometer; multivariate detection limits; sampling accessories; licorice; partial least squares regression.

\section{Introduction}

Most wet chemistry methods have been used for a long time, such as high-performance liquid chromatography (HPLC). These technologies usually have some disadvantages like tedious sample preparation procedures, time-consuming, labor-intensive and consumption of organic solvent. The near infrared (NIR) comes from overtones and combinations of the fundamental vibration of hydrogen bonds. ${ }^{1,2}$ Recently, NIR technology is recommended for reliability, fast acquisition and nondestruction. The advantages of NIR spectroscopy over other analytical techniques have increased its acceptance in agriculture, food, pharmaceuticals, chemical and oil industry. ${ }^{3-5}$

The growing demand has fostered the development of sensitive and expeditious NIR techniques, which were capable of determining low concentration (below 1000 parts per million (ppm)) of Chinese herbal medicine (CHM), such as Holographic Grating, Fourier Transform, Acousto-Optic Tunable Filter and micro-electro-mechanical systems (MEMS). However, important drawbacks of NIR technology, low spectral intensities and overlapping bands, result in high detection limit. ${ }^{4,6-8}$

The limit of detection (LOD) is significant values for analytical method assessment. The International Standards Organization (ISO) has recommended a LOD methodology to deal with analytical signal, which is a specific univariate. ${ }^{9,10}$ However, the ISO did not provide standard multivariate detection limits (MDL) method. NIR signals were complex and required multivariate calibration models. ${ }^{11-16}$ Therefore, an interesting point about MDL value using NIR technology has recently appeared in CHM system by our team, which is a significant parameter for NIR analysis of low concentration substance. ${ }^{17}$

Furthermore, MEMS-NIR is a new generation of optical spectrometer, which adopts a novel approach (microfabrication technology) to improve the measurement precision and sensitivity. In addition, sampling accessories have emerged with improved accuracy and precision by NIR spectrometer. ${ }^{18-21}$ Two sampling accessories, the FOP and the integrating sphere, are widely used in MEMS-NIR systems according to the guideline recommended by the manufacturer. ${ }^{22}$ In this work, based on the MEMS technology coupled with two sampling accessories, MDL estimator was used to assess the technique's detection limit for low-content quality parameters in CHM.

Glycyrrhizae Radix et Rhizoma, called as licorice, has been used in China for over 2000 years, which can be used to treat lassitude, severe palpitation, spleen and stomach weakness, etc. ${ }^{23}$ Licorice has a wide range of sources, including different areas, different production modes (wild or cultivate) and different cultivation conditions. In this paper, licorice in different geographical regions are used as samples, and partial least squares (PLS) was used to determine four quality parameters in licorice, glycyrrhizic acid, liquiritin, liquiritigenin and isoliquiritin. These quality parameters were recommended by Chinese Pharmacopoeia (2010 edition).

Chemometrics indicators, which are based on relative mean prediction errors of all concentration levels, i.e., root mean square error (RMSE), correlation coefficient $(R)$ and standard error of prediction (SEP), have been utilized to assess the model using MEMS-NIR technology coupled with two sampling accessories. Meanwhile, MDL estimator was utilized to assess the multivariate NIR model. ${ }^{24}$ The detection rule for MDL estimator was performed according to the Neyman-Pearson test, which considered alpha error and beta error: false positive and false negative. ${ }^{10,13,14}$

As mentioned in the previous report, ${ }^{17} \mathrm{MDL}$ estimator could evaluate prediction ability for low concentration. This research has shown that MDL estimator was utilized for MEMS-NIR technology, and it has never been reported before. In addition, reports about comparing the MDL estimator of diffuse reflection using two sampling accessories in the CHM system (i.e., licorice) had never been seen. Finally, based on the MDL result, an accurate 
replication for detection capability of four quality parameters in licorice was given based on the MEMS-NIR technology coupled with two sampling accessories.

\section{Materials and Methods}

\subsection{Materials}

Licorice was provided by Chinese Academy of Medical Science \& Peking Union Medical College (Beijing, China). These materials were collected from 24 different geographical regions in PR China, and were identified as the root and rhizome from Glycyrrhizia uralensis Fisch. by Prof. Wang Wenquan.

After cleaning by brushing off any soil dust, licorice was crushed into pieces by a disintegrator, then ground to fine pieces with a blender and screened through a 60 -mesh sieve. To avoid influences of water content, all powder samples were dried at $50^{\circ} \mathrm{C}$ for $24 \mathrm{~h}$ using an oven.

Liquiritin and glycyrrhizic acid were provided by National Institute for the Control of Pharmaceutical and Biological products (Beijing, China). Isoliquiritin and liquiritigenin were purchased from the Ronghe Medical Technology Co., Ltd (Shanghai, China). The purity of the four standards was above 98\%. Acetonitrile for HPLC grade was obtained from Merck (Darmstadt, Germany). Deionized water was prepared by Milli-Q purification system (Millipore Corp., USA).

\subsection{Preparation of solutions}

Each licorice powder was analyzed through NIR and HPLC analysis. Solutions were obtained by adding $100 \mathrm{mg}$ of licorice powder into a $100 \mathrm{~mL}$ amber volumetric flask. Licorice powder was sonicated for $30 \mathrm{~min}$ using $50 \mathrm{~mL}$ of $70 \%$ ethanol solvent, and then the solution was diluted to a volume of $100 \mathrm{~mL}$ with $70 \%$ ethanol solvent. These solutions were filtered through filter paper and further filtered by $0.45 \mu \mathrm{m}$ filter membrane directly into HPLC vials for immediate analysis.

\subsection{NIR equipment and two sampling accessories}

Axsun 410 Miniature MEMS-NIR Analyzer (Axsun Technologies, Billerica, MA, USA) was used to collect NIR spectra. Two sampling accessories, integrating sphere (Axsun Technologies, Billerica, MA, USA) and FOP (Axsun Technologies, Billerica, MA, USA) were used to obtain spectral data. The scan of 32 times and the resolution of $0.5 \mathrm{~nm}$ were performed to obtain the spectra. The spectrum of each licorice powder, in the range from 1350 to $1800 \mathrm{~nm}$, was analyzed three times. The average spectrum of licorice powder was used in the following analysis.

Licorice powder was divided into two parts (calibration set (56 samples) and prediction set (56 samples)) using Kennard-Stone (KS) method built in Matlab (MATLAB v 7.0, MathWorks, Massachusetts). Commercial software - Unscramble software (version 7.6, CAMO ASA, Oslo, Norway) was used to build PLS model based on cross-validation with a segment size of four.

\subsection{HPLC apparatus}

Licorice powder was extracted by KQ-500GDV ultrasonic bath $(250 \mathrm{~W}, 40 \mathrm{kHz})$ (KUNSHAN Ultrasonic, Jiangsu, China). The HPLC method for four quality parameters was performed by Agilent 1100 HPLC system (Palo Alto, CA, USA) and Waters Sunfire-C18 column $(150 \mathrm{~mm} \times 4.6 \mathrm{~mm}$ with $5 \mu \mathrm{m}$ particles, USA).

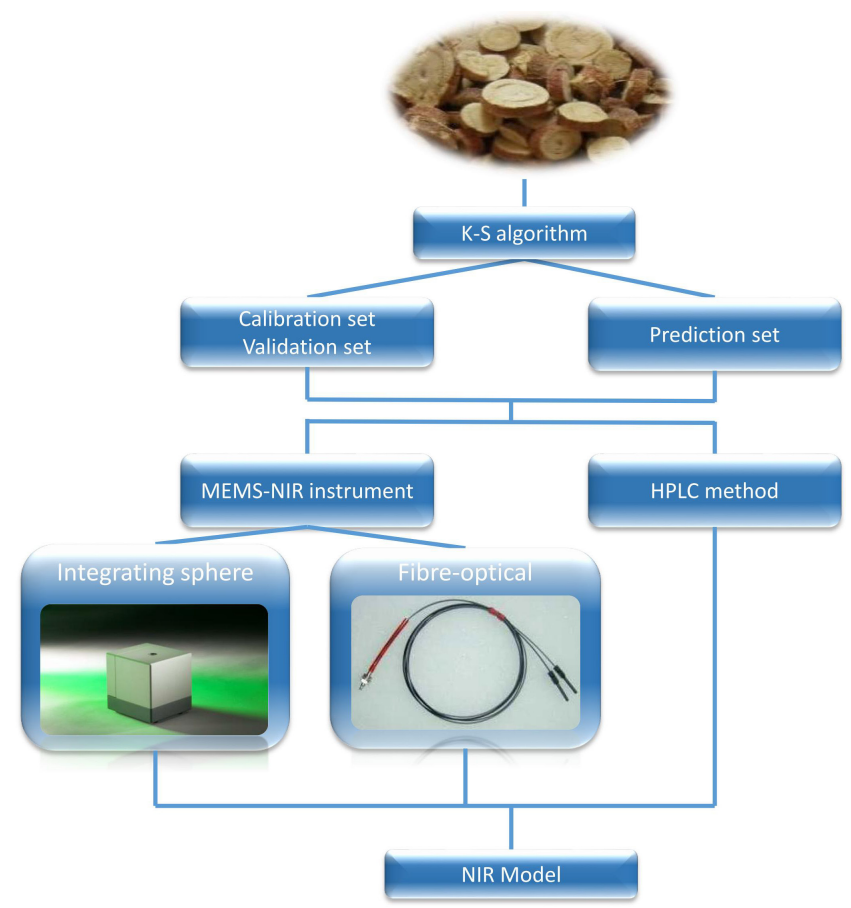

Fig. 1. The experimental protocol of NIR and HPLC analysis. 


\section{Z. Wu et al.}

Mobile phase consisted of (A) acetonitrile and (B) $0.05 \%$ phosphoric acid-water $(\mathrm{v} / \mathrm{v})$. The gradient program was used according to the following profile. Based on the initial 20\% (A), it was changed from $20 \%$ to $50 \%$ (A) in $25 \mathrm{~min}$. After $3 \mathrm{~min}$, it reached $100 \%$ of mobile phase $\mathrm{A}$, which was maintained for $5 \mathrm{~min}$. Then, the proportion of mobile phase A returned to $80 \%$ in 3 min. Finally, this proportion decreased to $20 \%$ in $5 \mathrm{~min}$. A flow rate of $1.0 \mathrm{~mL} / \mathrm{min}$ and column temperature at $25^{\circ} \mathrm{C}$ were maintained. $10 \mu \mathrm{L}$ of fluid was injected into the HPLC instrument. The DAD detector was operated at $248 \mathrm{~nm}$ (glycyrrhizin acid), $276 \mathrm{~nm}$ (liquiritin and liquiritigenin) and $370 \mathrm{~nm}$ (for the isoliquiritin), respectively. Figure 1 shows the experimental procedure according to the calibration (cross validation) and prediction sets.

\section{MDL Theory and Algorithm}

In this paper, the MDL theory is briefly described as follows: (1) analysis models; (2) LOD for model against alpha error; (3) LOD for model against alpha error and beta error; (4) MDL for model against alpha error and beta error. More descriptions about MDL theory and algorithm were reported in Refs. 10 and 13.

\subsection{Analysis models}

The model should be taken into account in formulating the LOD based on response $Y_{x}$ and concentration $X$. The model assumes that when $X=x$ :

$$
Y_{x}=\alpha+f_{(x, \beta)}+\varepsilon,
$$

where $\alpha$ is the parameter, which is corresponding with background value $Y_{0}$. The function $\left(f_{(x, \beta)}\right)$ is monotonic, in which the feature is $f_{(x 0, \beta)}=0 . \beta$ is a vector or parameter, and $\varepsilon$ is the measurement error for all $x$.

\subsection{LOD for model against alpha error}

According to Eq. (1), statistical approach about protection against alpha error is applied to the LOD. Protection against alpha error was denoted as "Assert $X>0$, if $Y>y_{P}$ ", where response $y_{p}$ is a selective threshold value.

Therefore, alpha error could be setup at a specified probability level, $p$. The model about protection against alpha error is calculated by Eq. (2).

$$
y_{P}=\alpha+\sigma Z_{p} / \sqrt{r}
$$

where $Z_{p}$ is the upper $100 * p$ (e.g., $\left.p=0.05\right)$ percentage point of the standard normal distribution; $\sigma$ is the constant variance; $r$ represents subsequent measurements (e.g., $r=1,2$, or 3 ).

\subsection{LOD for model against alpha error and beta error}

Furthermore, according to the statistical rule, beta error $(q)$ is performed for any $x>0$. The lowest concentration value $\left(x_{d,(p, q)}\right)$ is denoted as LOD, in which the detection rule is "Assert $X>0$, whenever $Y>y$ ". Thus, the function about $x_{d,(p, q)}$ is determined based on $r, p, q$ and other known model parameters, as shown in Eq. (3).

$$
x_{d,(p, q)}=\left(z_{p}+z_{q}\right) \sigma / \beta \sqrt{r}
$$

Alternatively, the formula is converted to Eq. (4). Thus,

$$
x_{d,(p, q)}=\omega_{0} \Delta_{(p, q)} \sigma / \beta,
$$

where $\Delta_{(p, q)}$ is the parameter of a noncentral Student's $t$ distribution with $\nu$ degrees of freedom, which take into account alpha error and beta error. $\Omega_{0}$ is defined as:

$$
\omega_{0}^{2}=\frac{1}{r}+\frac{1}{n}+\frac{\bar{x}^{2}}{Q_{x x}},
$$

$\bar{x}$ is the average (over all $n$ observations) of responses, $Q_{x x}$ is the covariance of $x$.

\subsection{MDL for model against alpha error and beta error}

Finally, MDL for multivariate model against alpha error and beta error can be calculated using Eq. (4) by an inverse calibration system. The MDL function is shown in Eq. (6).

$$
\mathrm{MDL}_{k}=\Delta_{(p, q)} \widehat{\operatorname{var}}\left(c_{0}, k\right)^{1 / 2},
$$

where the formula $\left(\widehat{\operatorname{var}}\left(c_{u m}\right)\right)$ is shown as:

$$
\left[(1+h) \mathrm{MSEC}-\bullet{ }_{c}^{2}\right],
$$

$h$ is the leverage value in calibration space of multivariate model. $k$ is the scalar factor ( 3 for MDL).

$\bullet_{c}^{2}$ is the concentration variance using HPLC method. MSEC parameter is the mean square error 
of calibration. Thus, MDL value can be calculated based on the MEMS-NIR technology coupled with two sampling accessories (integrating sphere and FOP).

\section{Results and Discussion}

\subsection{Determination of four quality parameters by HPLC method}

Four quality parameters content in licorice was determined using the method of Chinese Pharmacopoeia (2010 edition) and previous research of our team. ${ }^{25}$ Typical chromatograms of licorice extraction solution were shown in Fig. 2. Four quality parameters in the sample solution were identified on the basis of chromatographic retention time as compared to reference standard solutions.

The standard curves about four quality parameters are shown in Table 1. Good linearity about calibration curve was verified. This paper focuses on establishing an MEMS-NIR method of four quality parameters coupled with two sampling accessories. More detailed description about the chromatographic fingerprints and peak assignment was previously illuminated by Zhang and Ye. ${ }^{23}$

Furthermore, the statistical values of four quality parameters are shown in Table 2. For superior

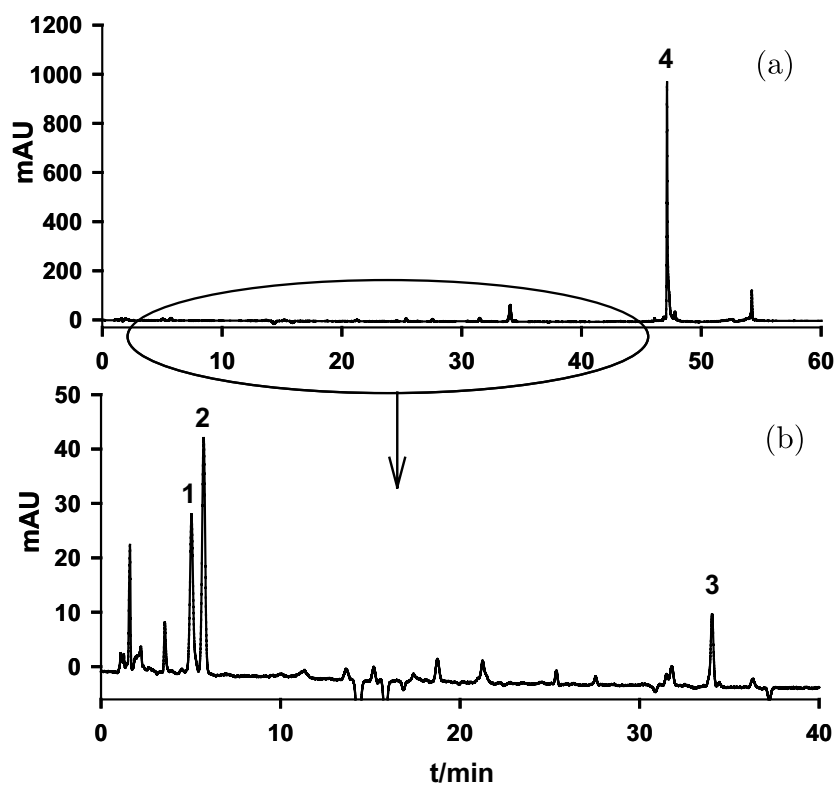

Fig. 2. Typical chromatograms of extraction solution, Peak 1 represents Liquiritigenin; Peak 2 represents Liquiritin; Peak 3 represents Isoliquiritin; Peak 4 represents Glycyrrhizic acid. advantage of this work, total representative samples are collected from genuine producing areas in China with the help of members in Engineering Research Center of Good Agriculture Practice for Chinese Crude Drugs of Ministry of Education.

The feature about calibration set consists of large concentration scale and concentration levels, which was suitable for establishing a robust calibration model. Then the goal of quantitative analysis for low analytes using MEMS-NIR technology was realized coupled with two sampling accessories, which has great value for the quality assessment of CHM greenly and rapidly.

\section{2. $\quad$ Spectral characteristic using MEMS technology coupled with two sampling accessories}

Figure 3 shows the NIR spectra using integrating sphere and FOP. As can be seen from Fig. 3, large fluctuations and baseline drifts appeared from raw spectra. For further comparison, MEMS-NIR technology using FOP generated more spectral glitch than the spectra of integrating sphere. Typically, the integrating sphere is slightly better than FOP.

\subsection{Comparison of different pretreatment methods using MEMS technology coupled with two sampling accessories}

Furthermore, the original spectra were processed for two sampling accessories. First derivative (1D) spectra and second derivative (2D) spectra show disorganized and unrecognized features. Due to derivative analysis, it showed that the spectral data of FOP were more susceptible to particle size effect, packing density and noise than data of integrating sphere (supporting information, Fig. 1s).

Pretreatment methods were applied to establish PLS models of MEMS spectra using two sampling accessories, i.e., 1D, 2D, standard normal variate (SNV), Savitzky-Golay algorithm (S.G. 9/2). The minimum RMSE value and $R^{2}$ was performed to select the optimum number of latent factors $s$ and the optimum processing method. The overall results show that each optimum pretreatment method and latent factors was selected differently according to the ingredient in licorice, i.e., S.G. 9 for liquiritin. 


\section{Z. Wu et al.}

Table 1. The calibration curves and LOD value of the HPLC method.

\begin{tabular}{lcccc}
\hline $\begin{array}{l}\text { Quality } \\
\text { parameters }\end{array}$ & Calibration curves & $R^{2}$ & Linearity ranges $(\mu \mathrm{g})$ & LOD $(\mathrm{ng})$ \\
\hline LIQG & $y=3 \times 10^{9} x+7282$ & 0.9990 & $\sim 1.77 \times 10^{-2}-0.106$ & 0.35 \\
LIQ & $y=3 \times 10^{9} x+28348$ & 0.9990 & $\sim 6.56 \times 10^{-2}-0.492$ & 0.82 \\
ISO & $y=5 \times 10^{9} x-25383$ & 0.9990 & $\sim 1.96 \times 10^{-2}-0.147$ & 0.49 \\
GLY & $y=6 \times 10^{8} x+33930$ & 0.9990 & $\sim 0.316-1.98$ & 3.95 \\
\hline
\end{tabular}

Note: LIQG, Liquiritigenin; LIQ, Liquiritin; ISO, Isoliquiritin; GLY, Glycyrrhizic acid.

Table 2. The statistics of four quality parameters in calibration and prediction sets.

\begin{tabular}{lccc}
\hline $\begin{array}{l}\text { Quality } \\
\text { parameters }\end{array}$ & $\begin{array}{c}\text { Average value } \\
\text { of concentration (\%) }\end{array}$ & $\begin{array}{c}\text { Concentration ranges } \\
\text { of calibration sets (\%) }\end{array}$ & $\begin{array}{c}\text { Concentration ranges } \\
\text { of prediction sets (\%) }\end{array}$ \\
\hline LIQG & 0.16 & $\sim 0.038-0.77$ & $\sim 0.049-0.62$ \\
LIQ & 1.36 & $\sim 0.072-4.39$ & $\sim 0.12-3.74$ \\
ISO & 0.26 & $\sim 0.024-1.02$ & $\sim 0.033-0.77$ \\
GLY & 3.90 & $\sim 0.52-10.74$ & $\sim 1.01-8.99$ \\
\hline
\end{tabular}

Note: LIQG, Liquiritigenin; LIQ, Liquiritin; ISO, Isoliquiritin; GLY, Glycyrrhizic acid.

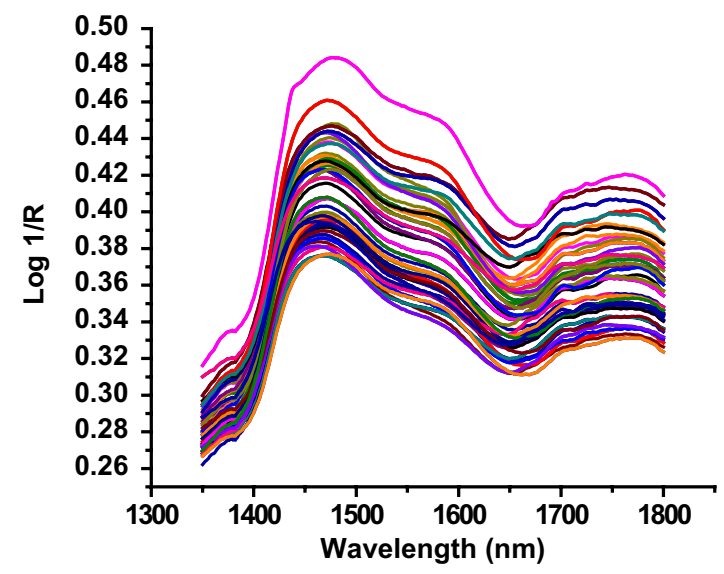

(a)

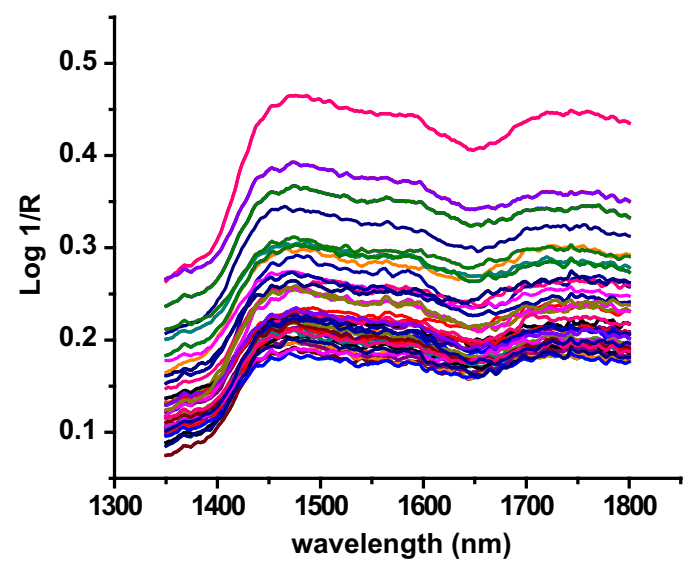

(b)

Fig. 3. NIR original spectra from integrating sphere (INS) and fiber optic probe (FOP), (a) raw spectra using integrating sphere, (b) raw spectra using FOP.

More detailed information is shown in the supporting information (Table 1s-8s).

\subsection{PLS model performance using MEMS technology for two sampling accessories}

The chemometrics indicators of all the models were listed in Table 3 and Fig. 4. For integrating sphere and glycyrrhizic acid, the number of factors was five.
The RMSE and $R$ were $0.173 \%$ and 0.9950 . The prediction gave Bias, SEP and $R$ value of 0.0259 , 0.3893 and $0.9877 \%$, respectively. Others can be observed in the same way. In general, the $R$ values of the calibration set and prediction set were all above 0.91 , and the values of BIAS and SEP were low, which indicated good detection ability using two sampling accessories. The results of the two sampling accessories show a perfect agreement between the MEMS-NIR prediction values and HPLC results. 
Table 3. Chemometrics indicators of all the PLS models for two sampling accessories (\%).

\begin{tabular}{|c|c|c|c|c|c|c|c|c|}
\hline \multirow[b]{2}{*}{ Accessories } & \multirow[b]{2}{*}{ Quality parameters } & \multirow[b]{2}{*}{ Spectral pretreatments } & \multirow[b]{2}{*}{ Latent factors } & \multicolumn{2}{|c|}{ Calibration set } & \multicolumn{3}{|c|}{ Prediction set } \\
\hline & & & & $R$ & RMSE & Bias & $R$ & SEP \\
\hline \multirow[t]{4}{*}{ INS } & GLY & $1 \mathrm{D}+\mathrm{SG}$ & 5 & 0.9950 & 0.173 & 0.0259 & 0.9877 & 0.3893 \\
\hline & LIQ & SG & 7 & 0.9923 & 0.082 & 0.0225 & 0.9765 & 0.1424 \\
\hline & LIQG & $2 \mathrm{D}$ & 7 & 0.9860 & 0.019 & 0.0050 & 0.9848 & 0.0169 \\
\hline & ISO & $2 \mathrm{D}$ & 6 & 0.9721 & 0.028 & 0.0039 & 0.9781 & 0.0353 \\
\hline \multirow[t]{4}{*}{ FOP } & GLY & $1 \mathrm{D}+\mathrm{SG}$ & 6 & 0.9879 & 0.268 & 0.1032 & 0.9755 & 0.4502 \\
\hline & LIQ & 1D & 7 & 0.9927 & 0.077 & 0.0472 & 0.9286 & 0.2638 \\
\hline & LIQG & $1 \mathrm{D}+\mathrm{SG}$ & 8 & 0.9963 & 0.010 & 0.0039 & 0.9844 & 0.0253 \\
\hline & ISO & 1D & 7 & 0.9938 & 0.014 & 0.0030 & 0.9954 & 0.0161 \\
\hline
\end{tabular}

Note: INS: integrating sphere; FOP: fiber optic probe; LIQ: Liquiritin; LIQG: Liquiritigenin; ISO: Isoliquiritin; GLY: Glycyrrhizic acid.

Furthermore, according to chemometrics indicators, the comparison result of two sampling accessories demonstrated that integrating sphere mode was more robust than FOP except for isoliquiritin content. Because fiber optic cables attenuate the signal as a function of distance, the longer fiber selections will have even lower signal-to-noise.

In addition, quality parameters in CHM presents low dose characteristics. Thus, a key question was the MDL of a calibration model. Furthermore, it was previously demonstrated that MDL was theoretically more accurate to assess model performance at low concentration levels than chemometrics indicators. ${ }^{17}$ Therefore, it was adopted in the following analysis.

\subsection{MDL estimator using MEMS technology for two sampling accessories}

Once each model was obtained, the MDL estimator was calculated using Eq. (6). Table 4 showed that MDL estimator was dependent on two types of errors (false positive and false negative). According to MDL estimator, different active components of the same spectrum have a large difference. When comparing the MDL estimator of all the PLS models, the result indicated that the accuracy and the precision of models using integrating sphere mode was higher than that of the FOP, which seemed to be owing to the additional interference information when FOP was introduced during sampling.
Spectral information reflected NIR rays within the acceptance angle using FOP. When the FOP is bent and moved, the angle of the MEMS-NIR ray may be less than the critical angle and the MEMSNIR ray is lost. This creates a change in the amount of energy that reaches the detector. In addition, integrating spheres collect rays reflected at any angle and also collect more diffuse reflectance from the sample. Therefore, taking both error $p(0.05)$ and error $q(0.05)$ calibration model as example, the MDL estimator of isoliquiritin is $0.01 \%$. Howerver, for glycyrrhizic acid, it is $1.28 \%$.

In detailed analysis, MDL estimator value depends on the " $(1+h)$ MSEC" and $\bullet 2$, which are used to calculate the $\omega$ parameter, as seen in Eq. (4). Based on the $\omega_{0}$ definition, it is explained that $\omega_{0}$ parameter presents characteristic of model performance at low concentration level (threshold value of response), rather than the prediction ability of one model based on mean prediction error of all concentration levels illustrated by chemometrics indicators.

Therefore, the MDL estimator results demonstrated that integrating sphere was a more powerful sampling accessory for obtaining high data quality. Overall, the results indicated that NIR spectroscopy combined with two sampling accessories could be successfully applied to determine the low content of glycyrrhizic acid, liquiritin, liquiritigenin and isoliquiritin in licorice. It clarified the MEMS-NIR detection ability in licorice coupled with two sampling accessories. However, this MDL formula neglects the errors in the NIR spectra of calibration 
Z. Wu et al.
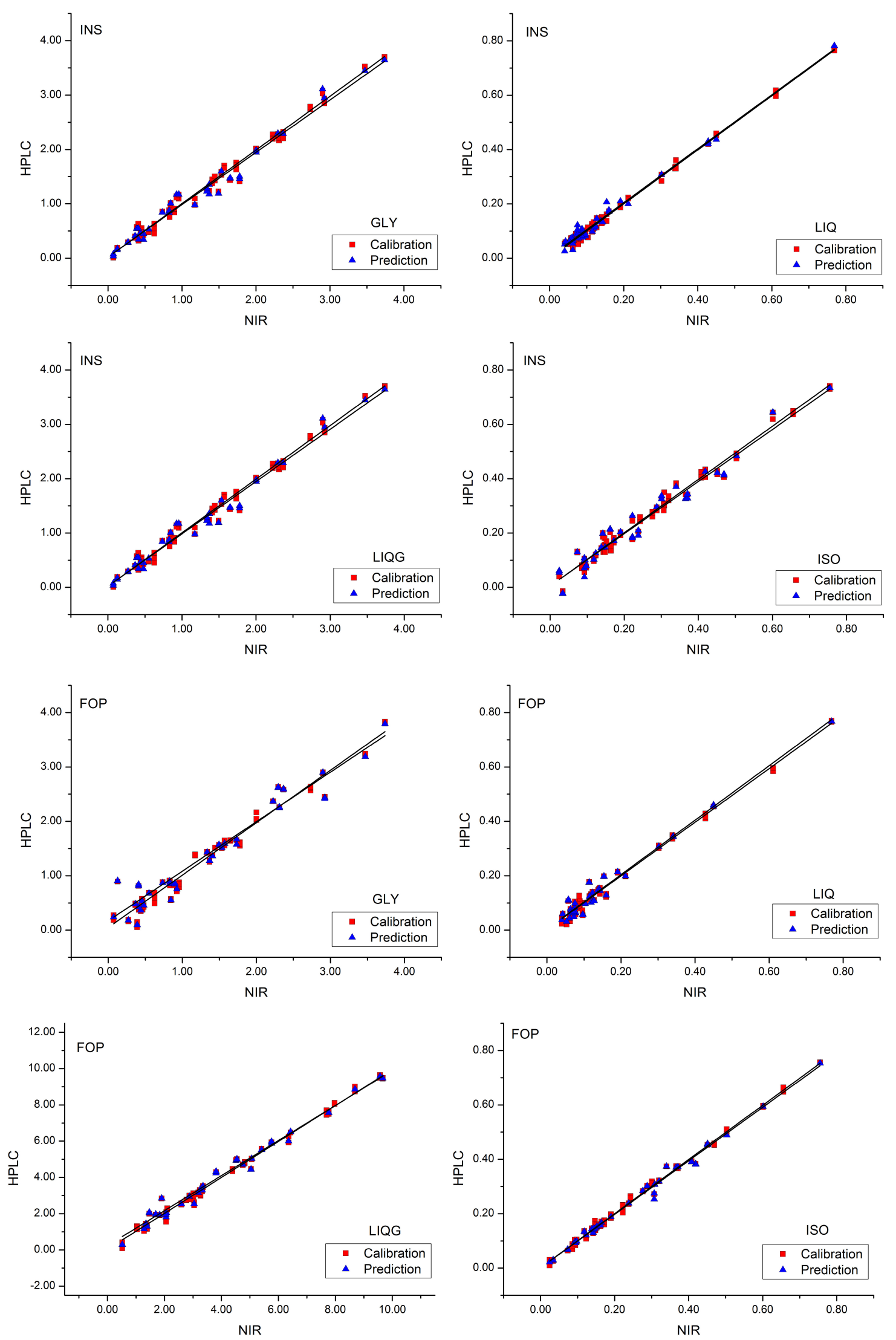

Fig. 4. Four quality parameters content of NIR predictions vs the reference method results. 
Estimation of $M D L$ of four quality parameters in licorice

Table 4. MDL estimator obtained from PLS model in two sampling technologies for different errors requirement (\%).

\begin{tabular}{lcccccccccc}
\hline Accessories & Quality parameters & $\Delta_{0.1,0.1}$ & $\Delta_{0.1,0.05}$ & $\Delta_{0.1,0.01}$ & $\Delta_{0.05,0.1}$ & $\Delta_{0.05,0.05}$ & $\Delta_{0.05,0.01}$ & $\Delta_{0.01,0.1}$ & $\Delta_{0.01,0.05}$ & $\Delta_{0.01,0.01}$ \\
\hline \multirow{2}{*}{ INS } & LIQ & 0.17 & 0.19 & 0.24 & 0.19 & 0.22 & 0.26 & 0.24 & 0.27 & 0.31 \\
& LIQG & 0.01 & 0.01 & 0.01 & 0.01 & 0.01 & 0.02 & 0.01 & 0.02 & 0.02 \\
& GLY & 1.0 & 1.13 & 1.40 & 1.14 & 1.28 & 1.55 & 1.41 & 1.56 & 1.82 \\
& ISO & 0.01 & 0.01 & 0.01 & 0.01 & 0.01 & 0.02 & 0.01 & 0.02 & 0.02 \\
FOP & LIQ & 0.38 & 0.43 & 0.53 & 0.43 & 0.48 & 0.58 & 0.54 & 0.59 & 0.69 \\
& LIQG & 0.01 & 0.02 & 0.02 & 0.02 & 0.02 & 0.03 & 0.03 & 0.03 & 0.03 \\
& GLY & 2.32 & 2.41 & 2.49 & 2.52 & 2.48 & 2.65 & 2.49 & 2.65 & 2.93 \\
& ISO & 0.02 & 0.02 & 0.03 & 0.02 & 0.02 & 0.03 & 0.03 & 0.03 & 0.03 \\
\hline
\end{tabular}

Note: INS: integrating sphere; FOP: fiber optic probe; LIQ: liquiritin; LIQG: liquiritigenin; ISO: isoliquiritin; GLY: glycyrrhizic acid.

set, which were pointed out by Lorber. ${ }^{26}$ In addition, the improvement of the methodology procedure should refer to mean square error of prediction (MSEP) parameter. We suggest that the further content should be improved for the MDL formula.

\section{Conclusion}

In this paper, MDL was used to evaluate the detection ability at low concentration level using MEMS-NIR coupled with two sampling accessories (integrating sphere and FOP). Four quality parameters in licorice from different geographical regions, glycyrrhizic acid, liquiritin, liquiritigenin and isoliquiritin, were determined by MEMS-NIR technology and PLS model. According to MDL estimator result, MEMS-NIR technology was able to detect minor analytes using integrating sphere and FOP. The detection ability of glycyrrhizic acid, liquiritin, liquiritigenin and isoliquiritin was separately determined in licorice using MDL estimator parameter.

In addition, the result suggests that PLS models using the integrating sphere mode have better generalization ability and prediction accuracy than FOP mode. The final result presented in this paper is not limited to give a guideline of MEMS-NIR about detection analysis in licorice, and could also help enormously in other chemical ingredients using diffuse reflection.

\section{Acknowledgments}

This work was financially supported from the National Natural Science Foundation of China (81303218), Doctoral Fund of China (20130013120006), Special
Fund of Outstanding Young Teachers and Innovation Team.

\section{References}

1. J. Workman, B. Lavine, R. Chrisman, M. Koch, "Process analytical chemistry," Anal. Chem. 83, 4557-4578 (2011).

2. J. Workman, M. Koch, B. Lavine, R. Chrisman, "Process analytical chemistry," Anal. Chem. 81, 4623-4643 (2009).

3. E. Rasanen, N. Sandler, "Near infrared spectroscopy in the development of solid dosage forms," $J$. Pharm. Biomed. 59, 147-159 (2007).

4. Y. Roggo, P. Chalus, L. Maurer, C. Lema-Martinez, A. Edmond, N. Jent, "A review of near infrared spectroscopy and chemometrics in pharmaceutical technologies," J. Pharm. Biomed. 44, 683-700 (2007).

5. J. Weeranantanaphan, G. Downey, P. Allen, D. W. Sun, "A review of near infrared spectroscopy in muscle food analysis: 2005-2010," J. Near Infrared Spec. 19, 61-104 (2011).

6. Z. S. Wu, M. Du, B. Xu, Z. Z. Lin, X. Y. Shi, Y. J. Qiao, "Absorption characteristics and quantitative contribution of overtones and combination of NIR: Method development and validation," J. Mol. Struct. 1019, 97-102 (2012).

7. W. F. McClure, "204 years of near infrared technology: 1800-2003," J. Near Infrared Spectrosc. 11, 487-518 (2003).

8. J. Luypaert, D. L. Massart, Y. V. Heyden, "Nearinfrared spectroscopy applications in pharmaceutical analysis," Talanta 72, 865-883 (2007).

9. M. Ostra, C. Ubide, M. Vidal, J. Zuriarrain, "Detection limit estimator for multivariate calibration by an extension of the IUPAC recommendations for univariate methods," Analyst 133, 532-539 (2008). 
10. R. Boque, N. K. M. Faber, F. X. Rius, "Detection limits in classical multivariate calibration models," Anal. Chim. Acta 423, 41-49 (2000).

11. G. Bauer, W. Wegscheider, H. M. Ortner, Fresenius, "Limits of detection in multivariate calibration," J. Anal. Chem. 340, 135-139 (1991).

12. A. Singh, "Multivariate decision and detection limits," Anal. Chim. Acta 277, 205-214 (1993).

13. R. Boque, F. X. Rius, "Multivariate detection limits estimators," Chemometr. Intell. Lab. Syst. 32, 1123 (1996).

14. R. Boque, M. S. Larrechi, F. X. Rius, "Multivariate detection limits with fixed probabilities of error," Chemometr. Intell. Lab. Syst. 45, 397-408 (1999).

15. M. Alcala, J. Leon, J. Ropero, M. Blanco, R. J. Romanach, "Analysis of low content drug tablets by transmission near infrared spectroscopy: Selection of calibration ranges according to multivariate detection and quantitation limits of PLS models," J. Pharm. Sci. 97, 5318-5327 (2008).

16. M. C. Sarraguca, J. A. Lopes, "The use of net analyte signal (NAS) in near infrared spectroscopy pharmaceutical applications: Interpretability and figures of merit," Anal. Chim. Acta 642, 179-185 (2009).

17. Z. S. Wu, C. L. Sui, B. Xu, L. Ai, Q. Ma, X. Y. Shi, Y. J. Qiao, "Multivariate detection limits of on-line NIR model for extraction process of chlorogenic acid from Lonicera japonica," J. Pharm. Biomed. 77, 1620 (2013).

18. D. Xiang, R. Lobrutto, J. Cheney, B. W. Wabuyele, J. Berry, R. Lyon, H. Q. Wu, M. A. Khan,
A. S. Hussain, "Evaluation of transmission and reflection modalities for measuring content uniformity of pharmaceutical tablets with near-infrared spectroscopy," Appl. Spectrosc. 63, 33-47 (2009).

19. R. S. Harner, R. J. Ressler, R. L. Briggs, J. E. Hitt, P. A. Larsen, T. C. Frank, "Use of a fiber-optic turbidity probe to monitor and control commercialscale unseeded batch crystallizations," Org. Process Res. Dev. 13, 114-124 (2009).

20. W. Y. Li, M. C. Johnson, R. Bruce, S. Ulrich, H. Rasmussen, G. D. Worosila, "Mass-balanced blend uniformity analysis of pharmaceutical powders by at-line near-infrared spectroscopy with a fiber-optic probe," Int. J. Pharm. 326, 182-185 (2006).

21. W. Kessler, D. Oelkrug, R. Kessler, "Using scattering and absorption spectra as MCR-hard model constraints for diffuse reflectance measurements of tablets," Anal. Chim. Acta 642, 127-134 (2009).

22. http://axsun.com/products/nir-spectroscopy.php

23. Q. Y. Zhang, M. Ye, "Chemical analysis of the Chinese herbal medicine Gan-Cao (licorice)," J. Chromatogr. A 1216, 1954-1969 (2009).

24. K. Faber, B. R. Kowalski, Fresenius, "Improved estimation of the limit of detection in multivariate calibration," J. Anal. Chem. 357, 789-795 (1997).

25. Chinese Pharmacopoeia Commission, Pharmacopeia of People's Republic of China, China Medical Science Press, Beijing (2010).

26. A. Lorber, "Error propagation and figures of merit for quantification by solving matrix equations," Anal. Chem. 58, 1167-1172 (1986). 